PROBLEMS OF ENGINEERING CYBERNETICS AND ROBOTICS • $2021 \bullet$ Vol. 75, pp. 59-70

p-ISSN: 2738-7356; e-ISSN: 2738-7364

https://doi.org/10.7546/PECR.75.21.07

\title{
Planning and Developing Techniques in Working | Within Distributed Systems for Wireless Gathering, Transferring and Manipulation of Information Streams
}

\author{
Krasimir Markov \\ Institute of Information and Communication Technologies \\ at the Bulgarian Academy of Sciences \\ Acad. Georgi Bonchev Str., bl. 21113 Sofia, Bulgaria \\ E-mail: krasikasi@abv.bg
}

\begin{abstract}
The article deals with the problems of processing of wireless information. It describes hardware implementation of distributed systems for wireless gathering, transferring and manipulation of information streams. The main stages of this process (coding, modulating and signal filtration) have been investigated thoroughly, the ones that form the electrical diagram controlled by EMC standards. Furthermore, in this article there are results shown from calculating the parameters of the RFID antenna.
\end{abstract}

Keywords: Electromagnetic compatibility (EMC), near-field communication (NFC near-field communication), Radio-frequency identification (RFID)

\section{Introduction}

The information and communication technologies behind the information systems today are an integral part not only at the workplace, but at the home and the smart homes [1]. Such systems can be represented as a set of interworking components that function together with the mutual goal of achieving a task. The information system is a concept that covers all forms of gathering, storing, extracting, manipulating and disseminating information. The presented results described in [2-7] are achieved by using wireless transfer of data. It is shown that the results can be successfully applied for radar target detection and in the existing 
communication network receivers making use of pulse signals. An example for two targets detection with antenna with beam synthesizing is presented in [8].

RFID (Radio-frequency identification) systems are information systems built towards wireless gathering of data from transponders (sensors, smart cards, etc.), also known as tags, and toward receiving data from applied systems for satisfying the specific information needs of the system. The RFID systems consist of three main components - a reader device, a transponder and an antenna tuned to the correct frequency in which the data is being transmitted and received. The most commonly used RFID frequencies are 11. From them, the top used ones are $125 \mathrm{kHz}, 13.56 \mathrm{MHz}$ and $2.45 \mathrm{GHz}$. On receiving the modulated signal, the reader uses the command protocol to demodulate and decode the incoming data. In the case of more than one transponder signal being received, techniques for identification without conflicts are then used. An example of that is keeping the reading for a small period of time, another one is to concurrently ask every single transponder. The data in a transponder can identify a certain object in construction, loads in transporting work, cars, animals or humans. RFID devices are used with great success in automated production lines and storage systems as well as for bank transactions (NFC). They can store data that can then be read by a base station or by handheld readers. These mobile databases provide personnel with their necessary information, eliminating the need of creating and maintaining a centralized database. In the development process the stored in the transponders information can be actualized in which way the database is also actualized with data for the product.

Wireless transfer of data is the transferring of digitally encoded information between two endpoint devices in a certain environment without losing any information. It is done by following the stages below:

a) Encoding of the data;

b) Modulating the data;

c) Filtering the signals;

d) Wireless transfer of signals (antenna projection);

e) Demodulating of the data;

f) Digital manipulation of the data (correct choice of a microcontroller with an appropriate firmware).

In developing a certain device, the hardware engineers do not always pay attention to the specification for electromagnetic compatibility, which leads to delays for the development process of a certain product, loss of financial assets in different aspects, mainly meaning more money spent for further tests in specialized laboratories.

For quality control (CE) a certain electronic device is put through a number of electromagnetic compatibility tests (EMC) regulated by BDC, [17]. Electromagnetic compatibility is the ability of a certain device to function to a 
satisfactory level in an electromagnetic environment, without creating noise and other electromagnetic issues into the correct working of another device in that same environment. The technical methods of suppressing, limiting and fully removing of these are: Filtration; Monitoring, and Grounding.

An average price for EMC tests is 10000 euro for a full test. In the case of inconsistencies with the norms, the device is analyzed, corrected and tested again with the same financial terms, such as money spent for business trips of employees and money spent for a repeat developing of new PCB originals.

\section{Hardware Development of an NFC Reader}

The digital manipulation of the data inside the reader is done through a previously chosen microcontroller (chosen due to the functional specifications of the system) that does the user application. The microcontroller communicates with a specialized integrated circuit (CLRC66302HN, [15]) via a serial peripheral interface (SPI).

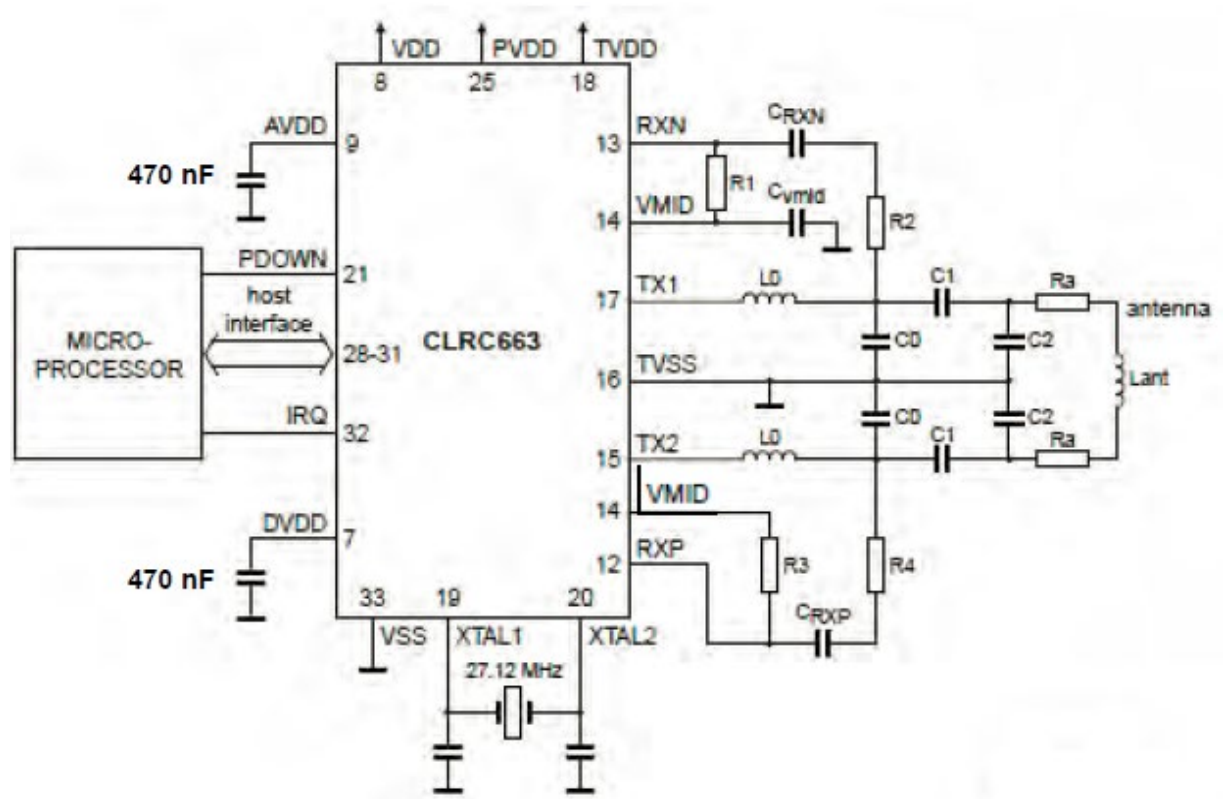

Fig. 1. NFC reader circuit

The RFID integrated circuit encodes the data received from the microcontroller using the SPI and uses them to modulate the carrying frequency $-13.56 \mathrm{MHz}$. The modulated signal is being transmitted to a filter to remove any high frequency harmonic signals that interfere in the environment and harm the working of other electrical components. After the filter is applied, the modulated 
signal is being passed through a post-tuning circuit that finalizes the tuning of the frequency of the signal, as well as synchronises it with an antenna (impedance and frequency). The circuit for this is shown in Fig. 1 and it is developed with the software product CAD STAR 16.

The NFC reader circuit shown in Fig. 1 contains: a microcontroller with a user application; an integral circuit CLRC66302HN [15]; an analog low-pass filter; and an analog circuit for synchronization of the impedance between it and the other part of the system (match circuit) [10].

\section{Theoretical Data and Examples for Calculating the Parameters of RFID Distributed System for Wireless Gathering, Transferring and Managing of Information Streams}

\subsection{Data Encoding}

The transferring of data is susceptible to noise from the environment or the channels, through which the data passes, including air. The electromagnetic noise, the interference and the fluctuations make the use of a defense necessary, one that defends against erroneous data transfer. The transfer process can be synchronous or asynchronous. The structuring of the stream of data is known as an encoding of the channel $[13,14]$. Here are some methods to do that (Fig. 2):

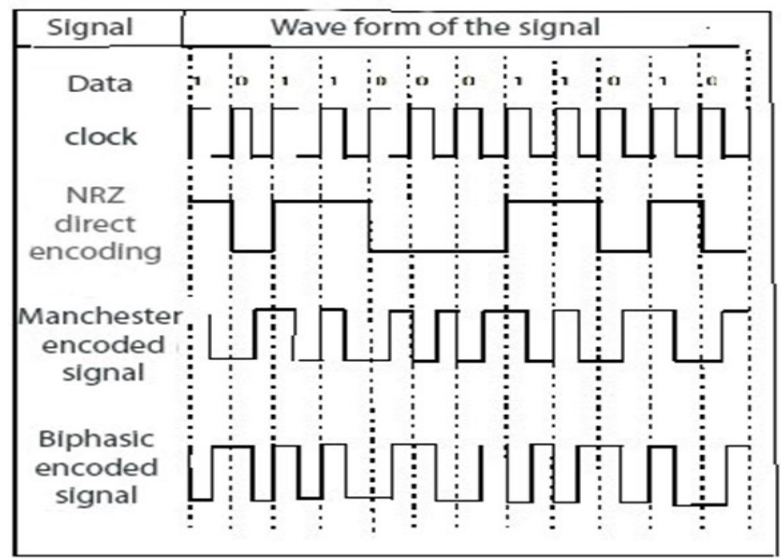

Fig. 2. Methods for signal encoding

NRZ is a direct method. In this case, the data is encoded depending on the period of the system clock, where a value of 0 is the low signal mode and a value of 1 translates to the high signal mode. Under Manchester encoding the change of signal level occurs for every half period of the clock. Biphasic encoding method is the same as a Manchester encoding but the phase of the output signal is rotated 
by 90 degrees. The data for this operation can be found in the description of integral circuit CLRC66302HN, [17].

\subsection{Data Modulation}

The transfer of data in the environment or the space between two components, that are exchanging information, requires it to be transformed into a fluctuating field or a wave. This transformation is called modulation [11]. Modulation is a process of changing the parameters of a signal, called a carrier signal, under the influence of another, called the modulating signal. The carrier signal has a much larger frequency than the modulating and is usually changed by the law of sines or cosines.

Depending on the parameter that is being modulated, modulation can be split into several types - amplitude modulation (AM), frequency modulation (FM), and phase modulation (PM). The last two are famous for their collective name angular modulation. When the carrier signal has rectangular form, an impulse modulation occurs. As with the other type, this can also be split into amplitude impulse modulation (AIM), frequency impulse modulation (FIM), phase impulse modulation (PIM) and wide impulse modulation (WIM). When the carrier signal is harmonic, and the modulating one has a rectangular form, the terms of manipulation are similar - amplitude, frequency and phase. The data for this operation can be found in the description of integral circuit CLRC66302HN, [17].

\subsection{Signal Filtering}

Development of the technical means of suppressing interference in the radio spectrum is done in this part. More information can be found in [11, 12]. On the next two figures (Fig. 3 and Fig. 4) the most common types of filters for this task can be found.

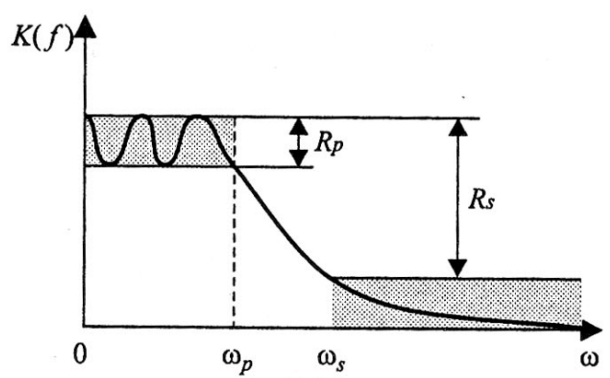

a) Low-freq. filter

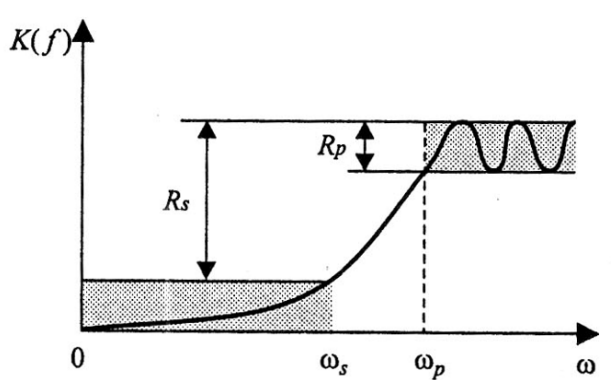

b) High-freq. filter

Fig. 3. Low and high-frequency filters 

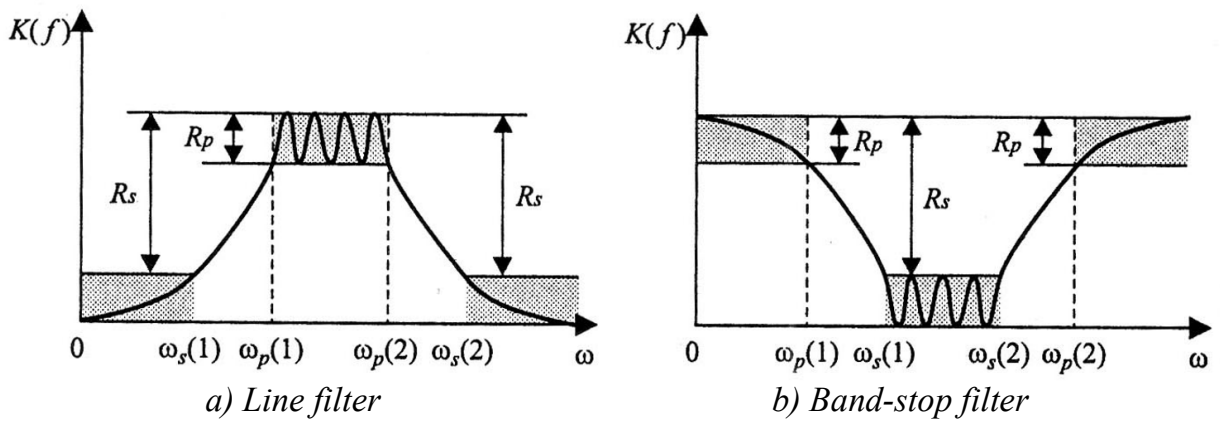

Fig. 4. Line and band-stop filter

\subsubsection{Method for calculating an EMC filter}

Due to the fact that integral circuit CLRC66302HN allows for the transferring of data through carrier frequency $13.56 \mathrm{MHz}$, the projection is limited to the development of a filter with passive components. The development of a filter with an operational amplifier is discouraged because the channel capacity is then less than or equal to $1 \mathrm{MHz}$ AND the cost of the system rises, $[11,12,16]$.

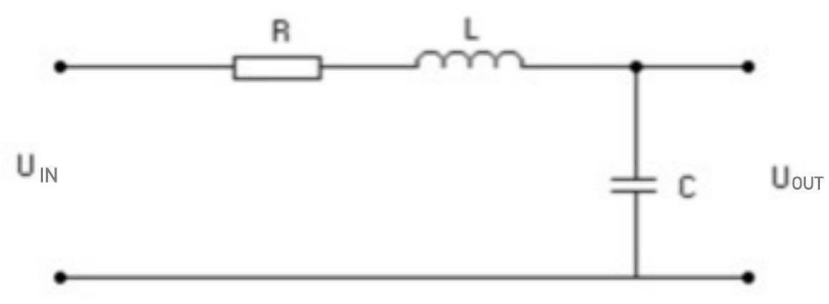

Fig. 5. Electrical circuit with passive elements

Transfer function of the filter can be represented as:

$$
W(p)=\frac{\frac{1}{\mathrm{pC}}}{\frac{1}{\mathrm{pC}}+\mathrm{pL}+\mathrm{R}}=\frac{1}{1+\mathrm{p}^{2} \mathrm{CL}+\mathrm{pCR}}
$$

Transfer function of a fluctuating track can be represented as:

$$
W(P)=\frac{k}{T^{2} p^{2}-2 \xi T p+1}
$$

The order of magnitude and the frequency of an environment are input parameters for the creation of a mathematical model for a transfer function for a filter. Some well-known polynomial approximations are those of Butterworth, Chebyshev, Leander (Papoolis) and by Bessel (Thompson). In the MATLAB environment, a mathematical model of a transfer function for Chebyshev is created as follows: 
Lo-pass filter Chebyshev

Wp $=2 * \mathrm{pi} * 20 \mathrm{e} 06$; $\%$ let through line

Ws $=2 * \mathrm{p} i * 25 e 06 ; \%$ suppress line

$\mathrm{Rp}=10$; $\%$ permissible unevenness of frequency response in the the suppression line in $\mathrm{dB}$

Rs=13; ㅇminimal necessary damping in the suppression line in $[\mathrm{n}, \mathrm{Wn}]=$ cheblord (Wp, Ws, Rp, Rs,'s') \%choosing of the minimal necessary order of magnitude of the filter $[\mathrm{b}, \mathrm{a}]=\mathrm{cheby}\left(\mathrm{n}, \mathrm{Rp}, \mathrm{Wn}, \mathrm{s}^{\prime}\right)$; ocalculating of an analog filter $h=t f(b, a) \circ t r a n s f e r$ function

The mathematical equation of the filter is:

$$
H=\frac{0.3162}{1.202 e-16 s^{2}+3.512 e-09 s+1}
$$

Using (3) it is possible to calculate the transfer function of the filter.

The poles of a characteristical polynomial are determined (the denominator of the transfer function). For a fluctuating track from the second order of magnitude there are two complex solutions with a real $(R E)$ and imaginary $(I M)$ part.

$$
\text { pol1 }=1.0 e+07 *(-1.4613 \mp 9.0051 i)
$$

$$
\begin{aligned}
& R E=-1.4613 \text { and } I M=9.0051 i . \\
& W_{c f}=\sqrt{R^{2}+I^{2}}=9.1229 e+07, f_{c}=2 . p i \cdot w_{c f}=14,52 \cdot 10^{6}
\end{aligned}
$$

$$
\begin{gathered}
\mathrm{T}=1 / W c f=1.0961 .10^{-8} \\
\zeta=\left(\operatorname{modul}\left(\frac{R}{W c f}\right)=0.1602\right.
\end{gathered}
$$

From equations (1), (2) and (3), the next relations can be written for determination of the optimal values for components in the electrical circuit:

$$
\begin{gathered}
\mathrm{T}^{2}=C * L \\
(2 * z * \mathrm{~T})=R * C
\end{gathered}
$$

For given inductivity $L=560 \mathrm{nH}$, we can calculate $C$ and $R$ that are $C=214,56 p F$ and $R=16 \Omega$.

\subsection{Sample Designing and Tuning of the NFC Antenna}

\subsubsection{Tuning of the NFC antenna}

In this part of the article, an object of interest is a circular NFC antenna, made as a flexible circuit board. The copper layer is placed between two layers of polyamide isolation, such as to stay in the neutral line of bending of the PCB. Measurements and construction of the base of the antenna have been presented below, using which the technical and electrical means are being calculated. The 
configuration method is also presented. It is used for wireless RFID communication.

The initial data of the parameters of a circular NFC antenna:

- $\mathrm{N}=2$ - number of coils;

- $\mathrm{r}=5 \mathrm{~cm}-$ distance that we want to transmit to;

- $\mathrm{a}=\sqrt{2} \cdot \mathrm{r}=7 \mathrm{~cm}-$ optimal coil radius;

- $\mathrm{b}=0.5 \mathrm{~cm}-$ thickness of the coil;

- $\mathrm{h}=0.5 \mathrm{~cm}-$ height of the coil.

The inductivity of the antenna is calculated using this formula

$$
L_{\text {ant }}=(a . N)^{2} \frac{0.31}{6 a+9 h+10 b}=1,2 \mu \mathrm{H}
$$

Where: $X_{L}=2 \pi f_{0 .} . L=100 \Omega$ is the induction resistance, $f_{0}=13.56 \mathrm{MHz}$ is the resonant frequency, $f_{\text {natural }}=520 \mathrm{MHz}$ is calculated using a $L C$ meter or a VectorImpedance Analyzer.

The condensator $C_{a n t}$ is calculated by the formula:

$$
C_{\text {ant }}=\frac{1}{\left(2 . \pi \cdot f_{\text {natural }}\right)^{2} \cdot L_{p a}}
$$

The condition when there is resonance in the circle is:

$$
X_{L}=X_{C}
$$

From condition (11) for the value of the adjusting capacitor we get:

$$
C=\frac{1}{2 \pi f 0 X L}=117 \mathrm{pF} \text { for } 13.56 \mathrm{MHz}
$$

The Quality factor $(Q)$ describes the energy, stored in the antenna. When the $Q$-factor is high, the antenna needs more time to react to the modulation but it is emitting more energy.

$$
Q \geq 13.56 \mathrm{MHz} * 3 \mu \mathrm{s}=40.68
$$

$Q=25$ is the standard value for NFC antennae.

The active resistance for the antenna $R$ is calculated using the formula:

$$
R=\frac{X L}{Q}=4 \Omega
$$

The gathered data from the analysis are summarized in Table 1.

Table 1. Table of gathered data from analysis

\begin{tabular}{|c|c|c|c|c|c|c|c|c|c|}
\hline$a$ & $b$ & $h$ & $r$ & $Q$ & $N$ & $L$ & $C$ & $R$ & $f_{0}$ \\
\hline $\mathrm{cm}$ & $\mathrm{cm}$ & $\mathrm{cm}$ & $\mathrm{cm}$ & - & - & $\mu \mathrm{H}$ & $\mathrm{pF}$ & $\Omega$ & $\mathrm{MHz}$ \\
\hline 14.14 & 0,5 & 0,5 & 50 & 25 & 2 & 1,2 & 117 & 4 & 13.56 \\
\hline
\end{tabular}




\subsubsection{NFC antenna adjustment}

Smith's diagram is meant to help with finding the solution to the problem with carrying lines and identical circuits [10]. Smith's diagram (Fig. 6) in essence is a graphical method to show the impedance of an antenna as a function of the frequency. It shows the complex coefficient of reflection, in a polar form for certain impedance. Meaning, we want to minimize the reflection coefficient, minimize the reflected power from the load (the antenna) and maximize the power that is received by the antenna, [9]. In order to reach the perfect balance of these three, we want the impedance of the load to be equivalent to the impedance of the carrying line. In the context of Smith's diagram, we wish to shift this impedance of the load towards the center where the reflection coefficient tends towards zero (Fig. 6).
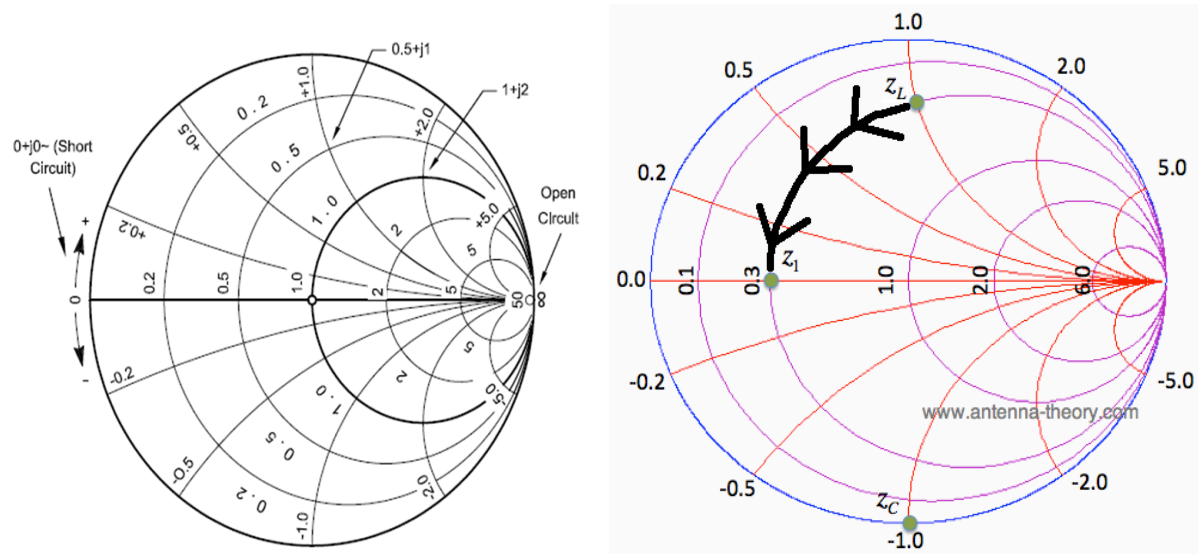

Fig. 6. Smith's diagram and the reflection coefficient

The circles describe the active (real) part of the impedance and the arcs the reactive (imaginary). The method for calculating a concurrence block is described in Fig. 7.

To calculate $Z_{t r}$ the following equation is used:

$$
Z_{t r}=R_{t r}+j \cdot X_{t r}
$$

where

$$
\begin{gathered}
R_{t r}=\frac{Z_{\text {match }}}{\left(1-\omega^{2} \cdot L_{0} \cdot C_{0}\right)^{2}+\left(\omega \cdot \frac{Z_{\text {match }}}{2} \cdot C_{0}\right)^{2}} \\
X_{t r}=2 \cdot \omega \cdot \frac{L_{0} \cdot\left(1-\omega^{2} \cdot L_{0} \cdot C_{0}\right)-\frac{z_{\text {match }}{ }^{2}}{4} \cdot C_{0}}{\left(1-\omega^{2} \cdot L_{0} \cdot C_{0}\right)^{2}+\left(\omega \cdot \frac{Z_{\text {match }}}{2} \cdot C_{0}\right)^{2}}
\end{gathered}
$$




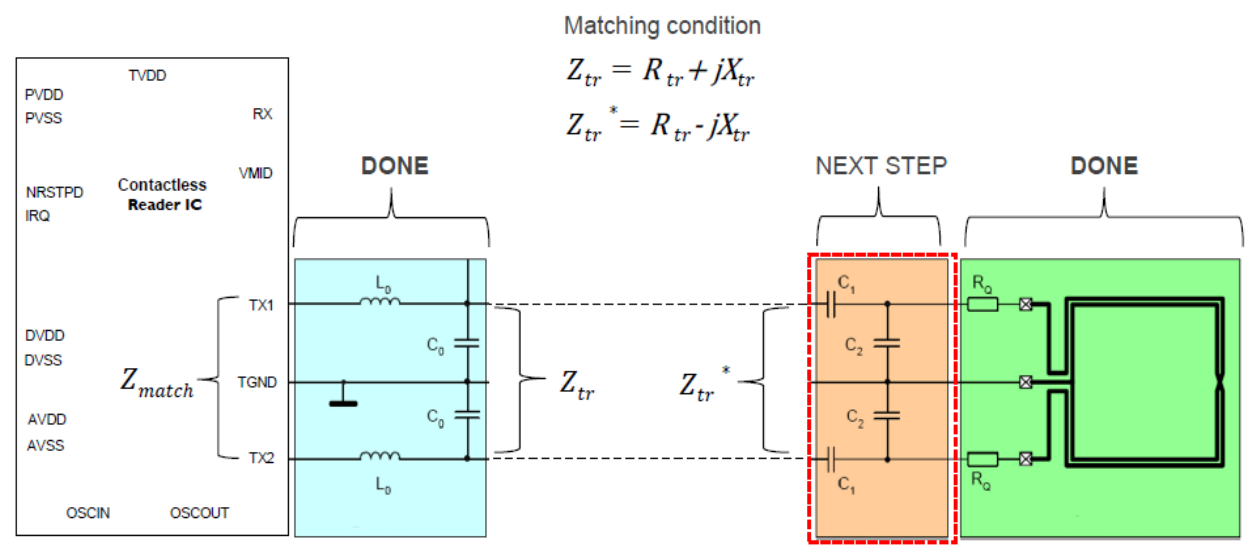

Fig. 7. Method for calculating a concurrence block

To calculate $Z_{t r}$ the following equation is used:

$$
Z_{t r}=R_{t r}+j \cdot X_{t r}
$$

where

$$
\begin{gathered}
R_{t r}=\frac{Z_{\text {match }}}{\left(1-\omega^{2} \cdot L_{0} \cdot C_{0}\right)^{2}+\left(\omega \cdot \frac{Z_{\text {match }}}{2} \cdot C_{0}\right)^{2}} \\
X_{t r}=2 . \omega \cdot \frac{L_{0} \cdot\left(1-\omega^{2} \cdot L_{0} \cdot C_{0}\right)-\frac{z_{\text {match }}{ }^{2}}{4} \cdot C_{0}}{\left(1-\omega^{2} \cdot L_{0} \cdot C_{0}\right)^{2}+\left(\omega \cdot \frac{z_{\text {match }}}{2} \cdot C_{0}\right)^{2}}
\end{gathered}
$$

The value of capacitor $C_{1}$ correcting coefficient of reflection towards zero is calculated using this formula:

$$
C_{1}=\frac{1}{\omega \cdot\left(\sqrt{\frac{R_{t r \cdot R_{\text {coil }}}}{4}+\frac{X_{\text {tr }}}{2}}\right)}
$$

The value of capacitor $C_{2}$ tuning the resonant frequency of the antenna is calculated using the formula:

$$
C_{2}=\frac{1}{\omega^{2} \cdot \frac{L_{a n t}}{2}}-\frac{1}{\omega \cdot \sqrt{\frac{R_{t r . R_{\text {coil }}}}{4}}}-2 . C_{\text {ant }}
$$

The parameters $C_{1}$ and $C_{2}$ are the elements of circuit referenced above. Then, this circuit is used to synchronize the impedance and frequency between the antenna and the rest of the system.

\section{Conclusion}

In this article, the results of the process of designing and developing the technical resources in implementing distributed systems for wirelessly gathering, transferring, and managing information streams are described. After establishing 
a baseline concept of the project, a system for wireless communication was developed, according to all NFC standards. The proposed system could be used for the identification of a user or a vehicle, access control, financial transactions, and the transfer of information with other NFC-based devices. Such particular applications are planned for future investigation.

\section{Acknowledgments}

This work was supported by the Bulgarian Ministry of Education and Science under the National Research Programme "Smart crop production" approved by Decision of the Ministry Council No 866/26.11.2020 (No D01-65/19.03.2021).

\section{References:}

1. Borissova, D., Dimitrova, Z., Garvanova, M., Garvanov, I., Cvetkova, P., Dimitrov, V., Pandulis, A.: Two-stage decision-making approach to survey the excessive usage of smart technologies, Problems of Engineering Cybernetics and Robotics 73, 3-16, (2020), https://doi.org/10.7546/PECR.73.20.01.

2. Doukovska, L.: Hough target detectors with small values of signal-to-noise ratio. NATO Advanced Study Institute "Unexploded Ordnance Detection and Mitigation", Il Ciocco, Italy, (2008).

3. Doukovska, L.: Combined Doppler-Hough method for velocity estimation. In: Proc. of the International Radar Symposium - IRS'09, Hamburg, Germany, pp. 677-682, (2009).

4. Doukovska, L., Angelova, D.: Comparative analysis of two techniques for moving target velocity estimation. In: Proc. of the 7-th European Radar Conference - EuRAD'10, Paris, France, pp. 431-434, (2010).

5. Doukovska, L.: Constant false alarm rate detectors in intensive noise environment conditions. Cybernetics and Information Technologies 10(3), 31$48,(2010)$.

6. Doukovska, L.: Application of mathematical transform in detection algorithms. In: Proc. of the First International Symposium on Business Modelling and Software Design - BMSD'11, Sofia, Bulgaria, pp. 161-167, (2011), DOI: $10.5220 / 0004459801610167$.

7. Doukovska, L.: Track-before-detect procedure using Hough velocity estimation technique. Comptes rendus de l'Academie bulgare des Sciences 68(9), 1153 $1160,(2015)$.

8. Doukovska, L.: Conventional Hough detector in presence of randomly arriving impulse interference. In: Proc. of the International Radar Symposium - IRS'15, Dresden, Germany, vol. 1, pp. 487-492, (2015).

9. Measurement and tuning of a NFC and Reader IC antenna - AN11535.

10. Oppenheim, Alan B., Allen C. Wilsky, Jan T. Young. Signals \& Systems. Prentice Hall, (1996).

11. Daniels, R. W.: Approximation Methods for Electronic Filter Design. McGrawHill, New York, (1974).

12. Zverev, A. I.: Handbook of Filter Synthesis. John Wiley, (1967). 
13. Ifeachor E., B. Jervis: Digital Signal Processing, A Practical Approach. Edinburg Gate: Pearson Education, (2002).

14. Penev, N.: Modern communication systems and technologies, Sofia, Nedkov, (2008).

15. NXP Semiconductors, CLRC663 - High performance multi-protocol NFC frontend CLRC663 and CLRC663 plus, (2020), https://www.mouser.bg/datasheet/2/302/CLRC663-1126414.pdf

16. Nikolov, E.: Technical means for automation, Parts 1 and 2, TU-Sofia, (2009).

17. Standards:

EMC for gauging noise emissions:

- Interfering voltage in the power outlets, frequency $150 \mathrm{kHz}-30 \mathrm{MHz}-\mathrm{BDS}$ EN 55011, BDS EN 55014-1, BDS EN 55015, BDS EN 55016-2-1

- Interfering voltage in the power outlets, frequency $30 \mathrm{MHz}-300 \mathrm{MHz}-$ BDS EN 55014-1

- Noise emission in the environment, frequency $30 \mathrm{MHz}-1 \mathrm{GHz}-\mathrm{BDS}$ EN 61000-4-20

- Noise emission in the environment, frequency $9 \mathrm{kHz}-30 \mathrm{MHz}-\mathrm{BDS}$ EN 55015

- Emission of radio-frequency disturbances in the frequency range $150 \mathrm{kHz}-$ $30 \mathrm{MHz}$ - BDS EN 55014-1

EMC regarding noise immunity:

- Immunity of electrical and electronic equipment to repetitive electrical fast transients. Voltage level $250 \mathrm{~V}-4000 \mathrm{~V}-$ BDS EN 61000-4-4

- Surge resistance. Voltage level $500 \mathrm{~V}-6000 \mathrm{~V}$ - BDS EN 61000-4-5

- Magnetic fields with frequency of the power network. Intensity of the magnetic field (0,5 - 150) A/m - BDS EN 61000-4-8

- Impulse magnet fields. Intensity of the magnetic field (0,5-150) A/m - BDS EN 61000-4-9

- EMV1 ISO/IEC 14443 Determines communication between reader and wireless smart cards. 